\title{
Frontières
}

\section{Charlotte Mémin, Comprendre la personne âgée, Paris, Bayard, 2001, 202 p.}

\section{Denise Badeau}

Volume 14, numéro 2, printemps 2002

URI : https://id.erudit.org/iderudit/1073984ar

DOI : https://doi.org/10.7202/1073984ar

Aller au sommaire du numéro

Éditeur(s)

Université du Québec à Montréal

ISSN

1180-3479 (imprimé)

1916-0976 (numérique)

Découvrir la revue

Citer ce compte rendu

Badeau, D. (2002). Compte rendu de [Charlotte Mémin, Comprendre la personne âgée, Paris, Bayard, 2001, 202 p.] Frontières, 14(2), 95-96.

https://doi.org/10.7202/1073984ar

Ce document est protégé par la loi sur le droit d'auteur. L'utilisation des services d'Érudit (y compris la reproduction) est assujettie à sa politique d'utilisation que vous pouvez consulter en ligne.

https://apropos.erudit.org/fr/usagers/politique-dutilisation/
Cet article est diffusé et préservé par Érudit.

Érudit est un consortium interuniversitaire sans but lucratif composé de l'Université de Montréal, l'Université Laval et l'Université du Québec à Montréal. Il a pour mission la promotion et la valorisation de la recherche. https://www.erudit.org/fr/ 
Charlotte Mémin

\section{Comprendre la personne âgée}

Paris, Bayard, 2001, 202 p.

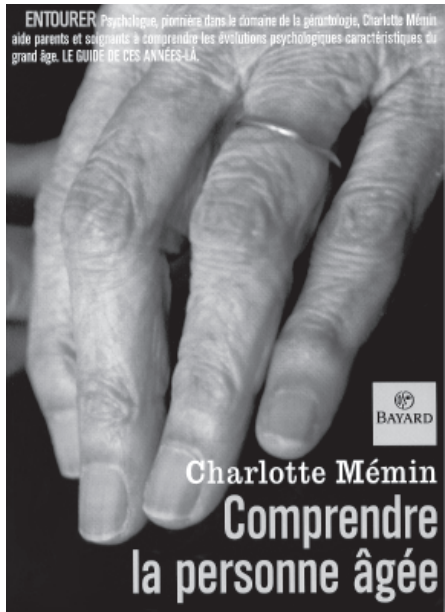

L'auteure de cet ouvrage considère la personne âgée dans sa globalité et elle se propose de répondre aux questions suivantes: existe-t-i une psychologie spécifique à la personne âgée? Comment communiquer avec cette dernière? Quels pièges éviter? Que devient la sexualité à mesure que l'on avance en âge? A-t-elle une place dans les institutions? Quelles attitudes adopter devant l'expression de la réalité sexuelle chez la personne âgée ?

Les réponses à ces questions ont donné naissance à quatre chapitres intitulés: psychologie de l'avance en âge, la communication, la sexualité, la transmission.

Le premier chapitre, le plus volumineux des quatre, tente de préciser ce qu'on entend par personne âgée. Tâche difficile, compte tenu de la réalité fort diverse et fort complexe sous-jacente au concept général et généralisant de personne âgée. Aussi pour parvenir à une définition plus exacte, faut-il, au préalable, préciser ce dont on parle: s'agit-il du vieillissement normal chez une personne d'âge avancé ou du vieillissement associé au handicap ou à la maladie? S'agit-il d'une personne âgée vivant à domicile, dans une maison de retraite ou en institution? Où est situé le lieu de vie ? À la campagne, à la ville ? Est-ce que la personne dont il s'agit vit seule, en couple ou en famille? Quels sont les services qui lui sont offerts? Autant de paramètres à préciser pour parvenir à une définition adéquate. L'auteure souligne la nécessité d'écouter la personne âgée pour mieux la connaître, pour savoir ce qui la soutient ou la déstabilise, la stimule ou l'appauvrit, la gratifie ou I'humilie (p. 23).

Après avoir affirmé que "le vieillissement est universel, inéluctable et différentiel » (p. 24), l'auteure identifie les principales causes de modification du comportement chez la personne âgée: les accrocs de santé, le décès d'êtres chers, les mutations de la structure familiale, la disparition d'un animal familier, la perte ou la destruction d'objets familiers, la ou les déceptions, l'entrée en institution particulièrement quand «ce changement de vie est décidé brutalement et arbitrairement » (p. 32), c'est-à-dire sans que la personne concernée n'ait eu vraiment son mot à dire dans la prise de cette grave décision.

Et d'ajouter l'auteure: «[...] reconnaissons que "le travail du vieillir" n'est pas aussi aisé qu'il y paraît. Ce parcours sinueux et chaotique est jonché de pièges, d'ambiguittés et de contradictions, d'angoisse et de sérénité, d'amertume et d'allégresse, d'assurance et de crainte, d'activité et de passivité, de repli sur soi et d'ouverture vers les autres " (p. 35).

L'auteure insiste sur le vieillissement du corps et l'influence de ce dernier sur le psychisme, sur la symbolique du corps selon l'investissement différentiel de ses différentes parties, sur l'angoisse de la mort et le deuil à effectuer, sur le rôle de l'environnement sur la santé mentale de l'individu âgé.

Le deuxième chapitre porte sur la communication, expression de la vie. Il identifie les principaux moyens de communication et définit le verbe communiquer. Jusque-là rien de très spécifique à la population à laquelle 
s'intéresse l'auteure. Cependant, une attention particulière est consacrée à la communication non verbale qui prend toute son importance chez la personne âgée privée de l'usage de la parole, ou aphasique, ou agnosique, chez la personne âgée malentendante, mal-voyante ou présentant des déficits cognitifs, ou dont la mémoire immédiate défaille.

Le troisième chapitre invite le lecteur à réfléchir sur la sexualité de la personne âgée. II distingue, bien que trop brièvement et trop vaguement, la sexualité de l'affectivité; puis, quelques données historiques amènent à considérer une connaissance plurielle de cette réalité. L'auteure souligne quelques événements susceptibles de moduler l'expression de la sexualité chez la personne âgée: la ménopause, le départ des enfants, la retraite... et consacre quelques pages à la sexualité dans les institutions gériatriques et à la sublimation, car « si le désir sexuel persiste tout au long de la vie, la fonction sexuelle perd de son intensité ou disparaît. C'est alors qu'intervient la sublimation » (p. 133), c'est-à-dire, selon le discours de certains psychanalystes, que « la pulsion sexuelle est dérivée vers un nouveau but non secret où elle vise des objets socialement valorisés» (p. 134): bénévolat, militantisme, vie intellectuelle, activité artistique.

La transmission fait l'objet du dernier chapitre. L'auteure y réitère sa conviction que la personne âgée, même diminuée, est une personne et que, comme telle, elle peut transmettre, communiquer, partager ce qu'elle a reçu, acquis, ce qui a nourri sa vie jusqu'à aujourd'hui. La personne âgée transmet des biens matériels: maison de famille, objets, archives... mais elle transmet aussi des souvenirs et des valeurs entendues comme « des principes qui donnent un sens à la vie» (p. 143). Comment peut-elle transmettre? Charlotte Mémin affirme " on transmet comme on transpire, par sa façon de vivre ". (p. 144) La personne âgée curieuse, réfléchie, capable d'étonnement, luttant contre l'engourdissement de ses facultés et l'emprise galopante d'un corps diminué, la personne âgée "en prise avec la vie " transmet ce qu'elle est, ce qu'elle a, ce qu'elle sait et ce qu'elle pense dans les échanges intergénérationnels grands-parents, petits-enfants, à la condition toutefois que chacun fasse son bout de chemin pour qu'advienne cette rencontre; les jeunes reconnaissant le grand-parent comme une personne vivante, sexuée, être de désir, capable encore de projets, les vieux réévaluant et interprétant leur existence à la lumière du présent. Les jeunes ont à apprendre des personnes âgées à « goûter un coucher de soleil comme on déguste un bon vin ", à « savourer la présence silencieuse d'un ami [...]" à "s'émerveiller du renouvellement des saisons comme de l'éclosion de l'amour [...] ", à " acquérir l'art de la mesure et celui du silence » (p. 176) et ce, dans l'amour et le respect réciproques.

D'une sensibilité indéniable à l'égard de la personne âgée, l'auteure de cet ouvrage a le mérite de présenter un contenu bien structuré, pertinent et sensé, appréhendant son objet d'étude dans sa globalité et elle le fait dans un discours simple, congruent, accessible, enrichi de nombreuses citations et d'exemples concrets appropriés facilitant la compréhension de la réalité de la personne âgée, objectif visé par l'auteure. Elle donne, à maintes reprises, tout au long du texte et particulièrement dans l'annexe, la parole à la personne âgée ellemême. Qui mieux qu'elle peut dire qui elle est et ce qu'elle vit? C'est un choix des plus judicieux. Bien que traitée de façon sommaire, imprécise et conservatrice, la sexualité des adultes âgés y est tout de même traitée, tâche dont peu d'auteurs et peu d'ouvrages s'acquittent quand ils abordent la vie de ces derniers.

Il a cependant le démérite de ne pas aller et de ne pas amener le lecteur à la découverte de sentiers inexplorés sur le vieillissement et la réalité de la personne âgée.

Toutefois, les professionnels auprès de la population âgée dans les services sociaux ou de santé, les personnes âgées elles-mêmes et les personnes avides de mieux les connaître, d'ici ou d'ailleurs, peuvent y trouver matière à réflexion.

Denise Badeau 